\title{
Ameliorate Effects of Li-Fu Formula on IL-6-Mediated Cardiac Hypertrophy in Hamsters Fed with a Hyper-Cholesterol Diet
}

\author{
Yi-Chang Cheng, ${ }^{1}$ Chieh-His Wu, ${ }^{2}$ Wei-Wen Kuo, ${ }^{2}$ James A. Lin, ${ }^{3}$ \\ Hsueh-Fang Wang, ${ }^{4}$ Fuu-Jen Tsai, ${ }^{5,6}$ Chang-Hai Tsai, ${ }^{7}$ Chih-Yang Huang, ${ }^{3,5,8}$ \\ Tsai-Ching Hsu, ${ }^{9,},{ }^{10}$ and Bor-Show Tzang ${ }^{10,11,12}$
}

${ }^{1}$ Emergency Department of Taichung Veterans General Hospital, China Medical University, Taiwan

${ }^{2}$ Department of Biological Science and Technology, China Medical University, Taiwan

${ }^{3}$ Graduate Institute of Basic Medical Science, China Medical University, Taiwan

${ }^{4}$ Department of Food and Nutrition, Huang-Kuang University, Taiwan

${ }^{5}$ Graduate Institute of Chinese Medical Science, China Medical University, Taiwan

${ }^{6}$ Department of Pediatrics, Medical Research and Medical Genetics, China Medical College Hospital, Taiwan

${ }^{7}$ Department of Healthcare Administration, Asia University, Taiwan

${ }^{8}$ Department of Health and Nutrition Biotechnology, Asia University, Taiwan

${ }^{9}$ Institute of Immunology, School of Medicine, Chung Shan Medical University, Taichung, Taiwan No. 110, Sec. 1, Jianguo N. Road, Taichung 402, Taiwan

${ }^{10}$ Clinical Laboratory, School of Medicine, Chung Shan Medical University Hospital, Taichung, Taiwan. No. 110, Sec. 1, Jianguo N. Road, Taichung 402, Taiwan

${ }^{11}$ Department of Biochemistry, School of Medicine, Chung Shan Medical University, Taichung, Taiwan. No. 110, Sec. 1, Jianguo N. Road, Taichung 402, Taiwan

${ }^{12}$ Institute of Biochemistry and Biotechnology, Chung Shan Medical University, Taichung, Taiwan. No. 110, Sec. 1, Jianguo N. Road, Taichung 402, Taiwan

Correspondence should be addressed to Bor-Show Tzang, bstzang@csmu.edu.tw

Received 11 March 2010; Accepted 13 May 2010

Copyright (C) 2011 Yi-Chang Cheng et al. This is an open access article distributed under the Creative Commons Attribution License, which permits unrestricted use, distribution, and reproduction in any medium, provided the original work is properly cited.

Hypercholesterolemia diets are considered as major sources to cause cardiac hypertrophy. This study intends to evaluate the effects of $\mathrm{Li}$-Fu formula on cardiac hypertrophy induced by hypercholesterolemia diet. Twenty-four male Golden Syrian hamsters were randomly divided into control, cholesterol and Li-Fu formula groups and fed with different experimental diets for 2 months. Histopathological analysis and western blotting were performed to measure the myocardial architecture, and various cardiac hypertrophy-associated molecules in the excised left ventricle from hamsters. The ratios of whole heart weight/body weight (BW) and left ventricle weight/BW were significantly higher in the cholesterol group but significantly lower in the Li-Fu formula group. The protein levels of both atrial natriuretic peptide and brain natriuretic peptide were significantly increased in the cholesterol group but significantly reduced in the Li-Fu formula group. Additionally, significantly increased interleukin-6, STAT3, MEK5, pERK5 and non-cardiomyocyte proliferate signal molecules such as p-MEK and p-ERK, were detected in the cholesterol group but significantly reduced in the Li-Fu formula group. Notably, no significant variations of inflammatory signaling molecules, including p-P38 and p-JNK, were detected in all groups. Our experimental results demonstrated the significant reductions of cardiac hypertrophy and related eccentric hypertrophy signaling, non-cardiomyocyte proliferate signaling in the excised left ventricle of hamsters from the Li-Fu formula. We suggested the protective effects of Li-Fu formula on cardiac hypertrophy that may be useful in prevention or treatment of hypertrophy-associated cardiovascular diseases.

\section{Introduction}

Hypercholesterol diets are the major cause of cardiac hypertrophy [1]. Cardiac hypertrophy is recognized as a cardiac adaptive response to any stress that can exist in a state of compensation or progress to a decompensated state over time [2]. Prolonged hypertrophy of the cardiomyocytes is demonstrated as the main cause of sudden cardiac death 
[3]. A number of studies indicated that various diseases have been associated with cardiac hypertrophy including occlusive atherosclerotic coronary heart disease (CHD), associated myocardial infarction (MI), heart failure hypertension, endocrine disorders, toxicants and bacterial endocarditis [47].

Atrial natriuretic peptide (ANP) and brain natriuretic peptide (BNP) are known as the cardiac hormones in normal adults that were secreted by the atria and ventricles. Higher levels of ANP and BNP expression are expressed in the fetal ventricles than adult ventricles [8]. Cardiac ANP and BNP levels are increased in MI of animal models [9], heart failure [10], hypertrophy [11] and also in human cardiac diseases [12]. Increased expressions of ANP and BNP are observed in ventricular during the molecular process of cardiac hypertrophy, which are recognized as markers of the induction of the embryonic gene program in ventricular hypertrophy [13].

Interleukin (IL)-6 is known as a potent hypertrophic factor of cardiomyocytes [14, 15]. The IL-6 receptor system consists of various signaling pathways including inflammatory-related p38 MAPK, hypertrophy involved STAT1-STAT3 heterodimer pathway, STAT3 homodimer pathway and non-cardiomyocyte proliferative related MAPK extracellular signal-regulated kinase (ERK)s pathway that are activated by the dimerization of gp130 [16-19]. The activation of STAT3-dependent signaling pathway by gp130 was reported to promote cardiac myocyte hypertrophy [20], herein the STAT1 and the STAT3 were shown to be chronically phosphorylated in the failing heart [21]. Moreover, the ERK5 molecule plays a critical role in postnatal eccentric hypertrophy of the heart $[9,22]$. ERK5 and its upstream MAPK-kinase 5 (MEK5) reveals a specific role in transduction of cytokine signals that regulate serial sarcomere assembly and in the induction of eccentric cardiac hypertrophy resulting in dilated cardiomyopathy and sudden death [22]. Therefore, it is crucial to investigate the pathologic role of IL-6-MEK5-ERK5 signaling pathway under cardiac hypertrophy. Additionally, various molecules have been elucidated responsible for the development of cardiac hypertrophy, including mitogen activated protein kinase (MAPK), phosphoinositide 3-kinase (PI3K) and calcineurin pathway [23]. The extracellular-regulated kinase (ERK), the c-Jun N-terminal kinases (JNK) and the p38 MAPK cascades (p38) enrolled in the MAPK pathway also play crucial roles in the development of cardiac hypertrophy [24].

To avoid the side effects by administration of western drugs, growing studies were performed to investigate the natural products for cardiac protection that have been used as drugs or diet supplements for a long history in many medical experiences. Recent studies reported the cardioprotective effect of various oriental herb extracts or dietary supplements including Fructus crataegi, Salvia miltiorrhiza and Astragali radix. The quercetin is the main ingredient in Fructus crataegi that has been demonstrated as an anti-inflammatory substance by inhibiting TNF- $\alpha$ release from macrophages and recognized to have cardiac protective effect $[25,26]$. Salvia miltiorrhiza is mainly composed of sodium tanshinone IIA sulfonate (STS), a derivative of tanshinone IIA that can reduce myocardial infarct size and prolong the survival cardiac cell in rabbit and human $[15,27,28]$. Astragali radix contains many isoflavones, isoflavonoids and many saponins, which have been demonstrated to have protective effects on heart by reducing inflammation, oxidant and cardiac ischemia-reperfusion injury [29-34] In our recent publication, we also demonstrated the protective effect of $\mathrm{Li}^{-}$ Fu formula composing of celery, black fungus, mushroom, Saliva miltior rhiza, Crataegi cuneata and Astragali radix on cardiac apoptosis [35].

To further understand the effects and possible mechanisms of Li-Fu formula on cardiac hypertrophy, we examined the expression of hyper-trophic associated molecules in the cardiac tissues from hamsters that were fed with hypercholesterol diets and suggested the cardiac protective effects of LiFu formula by reducing the cardiac hypertrophy.

\section{Methods}

2.1. Animals and Diet. Male Golden Syrian hamsters weighting $135-170 \mathrm{~g}$ at the age of 8 weeks were purchased from National Laboratory Animal Center, Taipei, Taiwan, and housed in an animal room at $22 \pm 2^{\circ} \mathrm{C}$ with a $12 / 12 \mathrm{~h}$ lightdark cycle under supervision of Institutional Animal Care and Use Committee of China Medical University, Taichung, Taiwan. Hamsters were acclimatized for 2 weeks while receiving free access to water and were fed chow diet (Lab Diet 5001; PMI Nutrition International Inc., Brentwood, MO, USA) ad libitum. For each experiment, 24 hamsters were randomized into three groups as control, cholesterol and $\mathrm{Li}-\mathrm{Fu}$ formula groups and switched to experimental diets. Three independent experiments were performed. The control, cholesterol and $\mathrm{Li}$-Fu formula groups received chow diet, chow diet with $0.2 \%$ cholesterol (Sigma, St Louis, MO, USA), and chow diet with $0.2 \%$ cholesterol and $2 \% \mathrm{Li}-\mathrm{Fu}$ formula for 8 weeks, respectively. Celery and Black fungus were obtained from common supermarket and mushroom, Saliva miltior rhiza, Crataegi cuneata and Stragali radix were purchased from traditional Chinese pharmacy. To make LiFu formula, every component of desired weight was crushed and mixed with a blender, then placed in $1000 \mathrm{ml}$ distilled water and boiled for $1 \mathrm{~h}$ under reflux. The resultant solution was divided into several parts and stored in a $-80^{\circ} \mathrm{C}$ freezer for further use [35]. The Li-Fu formula is composed of celery, black fungus, mushroom, Saliva miltior rhiza, Crataegi cuneata and Astragali radix as shown in Table 1 and the experimental dietary composition is shown in Table 2. Diets were prepared weekly and stored at $-80^{\circ} \mathrm{C}$. All experimental procedures were performed according to the NIH Guide for the Care and Use of Laboratory Animals. All protocols were approved by the Institutional Animal Care and Use Committee of China Medical University, Taichung, Taiwan. Food intake and food spillage were measured daily, and body weight was recorded every 3 days.

2.2. Cardiac Characteristics. Three groups of hamsters at age of 8-9 months were weighed and decapitated after receiving 8 weeks of experimental diets. The hearts of animals were 

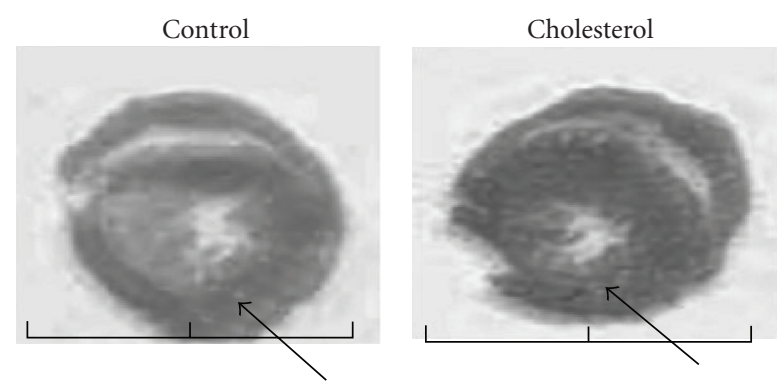

(a)
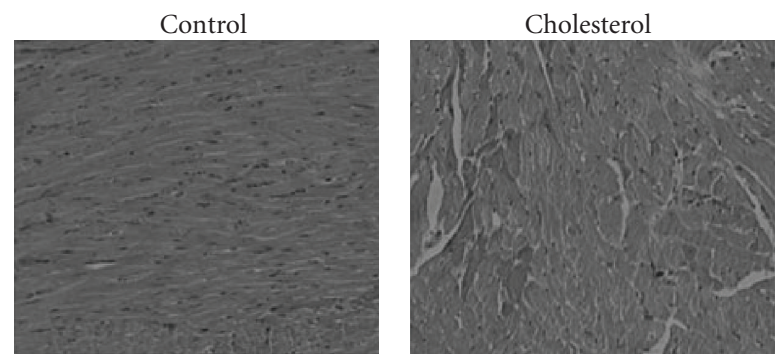

Li-Fu formula

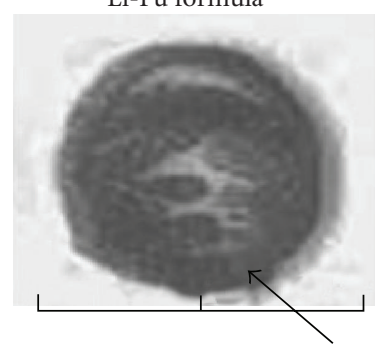

Li-Fu formula

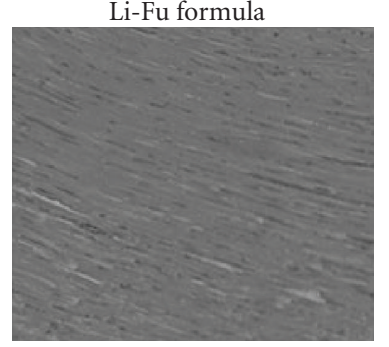

(b)

Figure 1: Cardiac cross sections and cardiomyopathic changes in hamsters of control, cholesterol and Li-Fu formula groups. (a) The cross section of whole heart in the three groups. Arrows indicate that the left ventricular lumen diameters increased in the cholesterol group but decreased in the Li-Fu formula group. (b) Representative histopathological analysis of cardiac tissue sections with hematoxylin and eosin staining in hamsters of control, cholesterol and Li-Fu formula groups. The images of myocardial architecture were magnified by 100 times.

TABle 1: Composition of Li-Fu formula.

\begin{tabular}{lcc}
\hline Items & Weight $(\mathrm{g})$ & Portion $(\%)$ \\
\hline Celery $^{(\mathrm{a})}$ & 25 & 2.5 \\
Black fungus $^{(\mathrm{b})}$ & 320 & 32.3 \\
Mushroom $^{(\mathrm{c})}$ & 420 & 42.4 \\
Saliva miltior rhiza $_{\text {Crataegi cuneata }}$ & 75 & 7.6 \\
Stragali radix & 75 & 7.6 \\
\hline
\end{tabular}

(a) Celery is also kanown as Apium graveolens.

(b) Black fungus indicates Wood ear, or pinyin: mù èr, lit. "wood ear" or "tree ear" are commonly sold in Asian markets as dietary supplement.

(c) The standard for the name "mushroom" is the cultivated white button mushroom, Agaricus bisporus.

TABLE 2: Formulation and calculated composition of diets.

\begin{tabular}{llll}
\hline $\begin{array}{l}\text { Ingredients }(\% \\
\text { wt/wt) }\end{array}$ & Control diet & $\begin{array}{l}\text { Cholesterol } \\
\text { diet }\end{array}$ & $\begin{array}{l}\text { Experimental } \\
\text { diet }\end{array}$ \\
\hline $\begin{array}{l}\text { Chow diet, } \\
\text { (Rodent 5001) }\end{array}$ & 99.5 & 99.3 & 97.3 \\
Soybean oil & 0.5 & 0.5 & 0.5 \\
Cholesterol & 0 & 0.2 & 0.2 \\
Li-Fu formula & 0 & 0 & 2 \\
\hline
\end{tabular}

excised and cleaned with distilled $\mathrm{H}_{2} \mathrm{O}$. The left and right atrium and ventricle were separated and weighed. The BW, left ventricle weight (LVW), the ratios of the whole heart weight (WHW) to BW and the ratios of the LVW to BW, were measured and calculated.

2.3. Hematoxylin-Eosin Staining. The hearts of animals were excised and were soaked in formalin and covered with wax. Slides were prepared by deparaffinization and dehydration. They were passed through a series of graded alcohols (100, 95 and 75\%), 15 min each. The slides were then dyed with hematoxylin. After gently rinsing with water, each slide was then soaked with $85 \%$ alcohol, $100 \%$ alcohol I and II for $15 \mathrm{~min}$ each. At the end, they were soaked with Xylene I and Xylene II. Photomicrographs were obtained using Zeiss Axiophot microscopes.

2.4. Tissue Extraction. Cardiac tissue extracts were obtained by homogenizing the left ventricle samples in a PBS buffer $\left(0.14 \mathrm{M} \mathrm{NaCl}, 3 \mathrm{mM} \mathrm{KCl}, 1.4 \mathrm{mM} \mathrm{KH}_{2} \mathrm{PO}_{4}, 14 \mathrm{mM}\right.$ $\mathrm{K}_{2} \mathrm{HPO}_{4}$ ) at a ratio of $100 \mathrm{mg}$ tissue $/ 0.5 \mathrm{ml}$ PBS for $5 \mathrm{~min}$. The homogenates were placed on ice for $10 \mathrm{~min}$ and then centrifuged at $12000 \mathrm{~g}$ for $30 \mathrm{~min}$. The supernatant was collected and stored at $-70^{\circ} \mathrm{C}$ for further experiments. Protein concentration was determined using a BioRad Protein Assay (BioRad Laboratories, Hercules, CA, USA) and were quantified by absorbance at $595 \mathrm{~nm}$ using a spectrophotometer (Beckman Coulter, Palo Alto, CA, USA).

2.5. Western Blot. Western blotting was performed as described in our previous report [35]. Protein samples were separated in $12.5 \%$ of SDS-PAGE and electrophoretically 


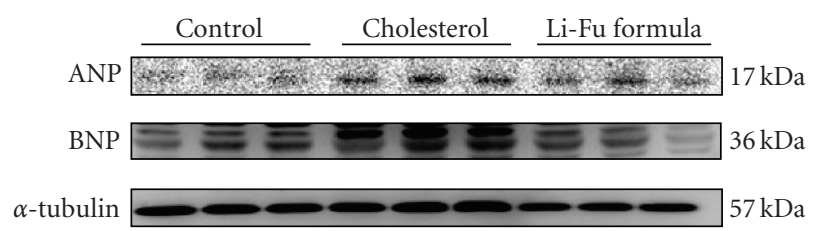

(a)

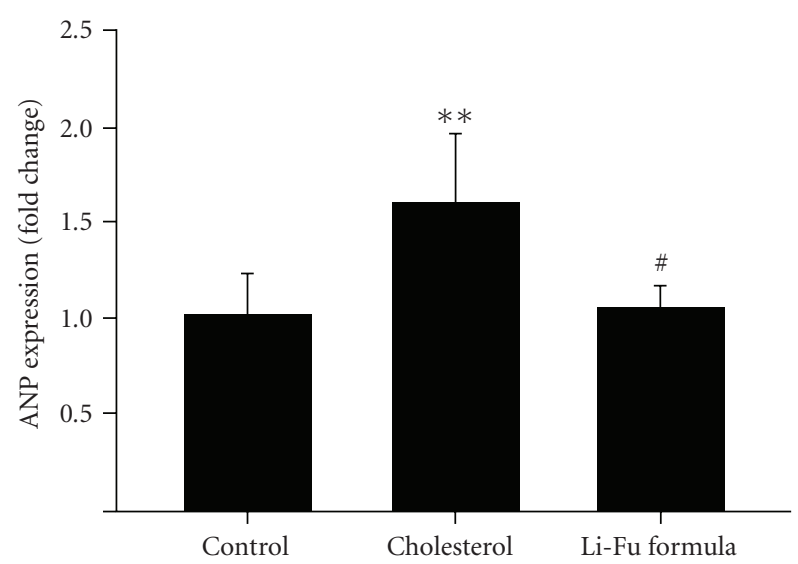

(b)

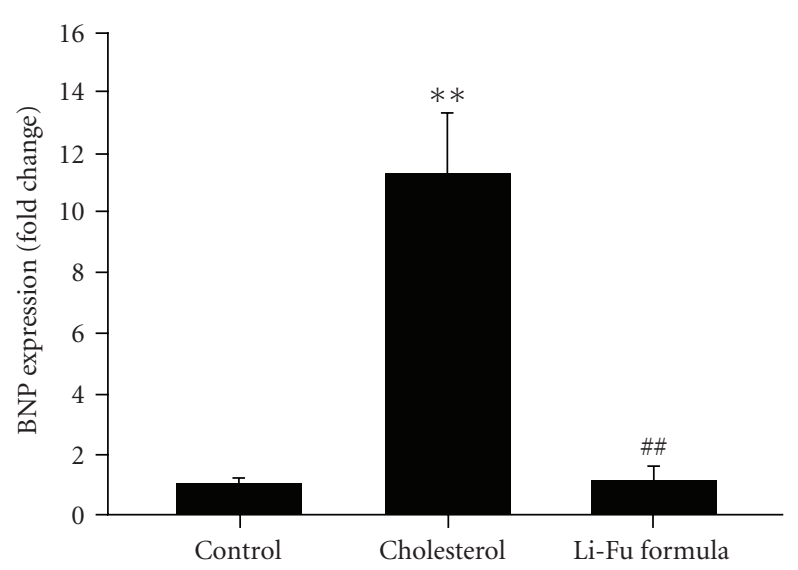

(c)

Figure 2: (a) The representative protein products of ANP and BNP extracted from the left ventricles of excised hearts in hamsters of control, cholesterol and $\mathrm{Li}-\mathrm{Fu}$ formula groups were measured by western blotting analysis. ((b), (c)) Bars represent the relative protein quantification of ANP and BNP on the basis of $\alpha$-tubulin. All bars indicate mean values $\pm \mathrm{SD}$ ( $n=6$ in each group). ${ }^{* *} P<$ .01 , significant differences between control and cholesterol group. ${ }^{\#} P<.05$ and ${ }^{\# \#} P<.01$, significant differences between cholesterol and Li-Fu formula groups.

transferred to nitrocellulose membrane (Amersham Biosciences, Piscataway, NJ). After blocking with 5\% non-fat dry milk in PBS for $30 \mathrm{~min}$ at room temperature, antibodies against ANP, BNP, IL-6, STAT3, MEK5, p-EKR5, MEK, pMEK, phosphorylated ERK (p-ERK), p-P38, JNK, p-JNK and $\alpha$-tubulin (Santa Cruz Biotechnology, Santa Cruz, CA, USA) were diluted to $1: 500$ with $5 \%$ nonfat dry milk in PBS and then incubated for $1.5 \mathrm{~h}$ at room temperature. The
TABLE 3: WHW, LVW, WHW to BW, and LVW to BW ratio.

\begin{tabular}{llll}
\hline & Control & Cholesterol & Li-Fu formula \\
\hline $\begin{array}{l}\text { Whole heart weight } \\
(\mathrm{mg})\end{array}$ & $410.6 \pm 36.10$ & $423.1 \pm 21.92$ & $377.1 \pm 2.40$ \\
LVW $(\mathrm{mg})$ & $290.5 \pm 8.82$ & $292.0 \pm 12.98$ & $269.1 \pm 10.10$ \\
WHW/BW $^{(a)}$ & $3.8 \pm 0.03$ & $3.9 \pm 0.01^{*}$ & $3.6 \pm 0.03^{\#}$ \\
LVW/BW $^{(a)}$ & $2.7 \pm 0.04$ & $2.8 \pm 0.01^{*}$ & $2.6 \pm 0.03^{\#}$ \\
\hline
\end{tabular}

(a) Data present in percentage.

${ }^{*} P<.05$, versus control.

${ }^{\#} P<.05$, versus cholesterol.

membranes were washed twice with PBS-Tween for $1 \mathrm{~h}$ and incubated with second antibody conjugated with horseradish peroxidase (Promega Corp., Madison, WI, USA) for $1 \mathrm{~h}$ that was diluted 1000-fold with 5\% nonfat dry milk in PBS. Pierce's Supersignal West Dura HRP Detection Kit (Pierce Biotechnology Inc., Rockford, IL) was used to detect antigenantibody complexes. The blots were scanned and quantified by densitometry (Appraise, Beckman-Coulter, Brea, CA, USA).

2.6. Statistical Analysis. All of the statistical analyses were performed using SPSS 10.0 software (SPSS Inc., Chicago, IL). Three independent experiments were repeated. Statistical analyses were performed using the analysis of variance plus posterior multiple comparison test to test the difference. The data between two experimental animal groups was compared by Student's $t$-test for two independent samples. In all cases, a difference at $P<.05$ was considered statistically significant.

\section{Results}

3.1. Experimental Diets and Cardiac Characteristics. To investigate the effect of Li-Fu formula on hypertrophy in cardiac cells, we examined the BW and cardiac characteristics. First, Li-Fu formula was prepared as described in materials and methods and the compositions of the Li-Fu formula was shown in Table 1. Table 2 presents the ingredients of experimental diets for different groups of hamsters. BW, LVW, the ratios of WHW to BW and the ratios of LVW to BW of hamsters from control, cholesterol and Li-Fu formula groups were detected (Table 3 ). The ratios of WHW/BW and LVW/BW were significantly higher in hamsters of cholesterol group compared to control group. Notably, the ratios WHW/BW and LVW/BW were significantly reduced in the hamsters from $\mathrm{Li}-\mathrm{Fu}$ formula group compared with cholesterol group (Table 3 ).

3.2. Cardiac Architecture Changes. To further confirm the effect of $\mathrm{Li}-\mathrm{Fu}$ formula on cardiac hypertrophy, we performed cross-sectional analysis of whole heart and histopathological analysis of ventricular tissue stained with hematoxylin and eosin. We found that ventricular wall thickness significantly increased in the cholesterol group, but decreased significantly in the Li-Fu formula group (Figure 1(a)). The ventricular myocardium in control group showed normal architecture with normal interstitial space. 


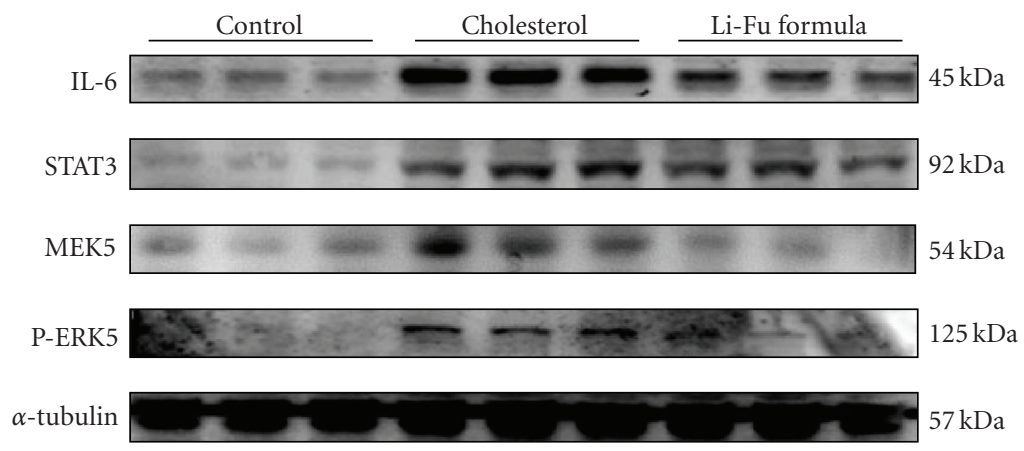

(a)

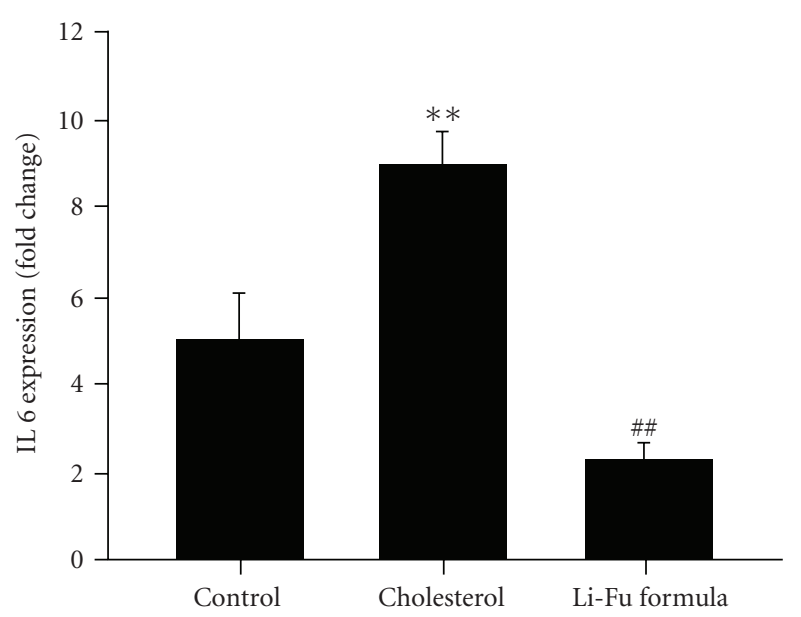

(b)

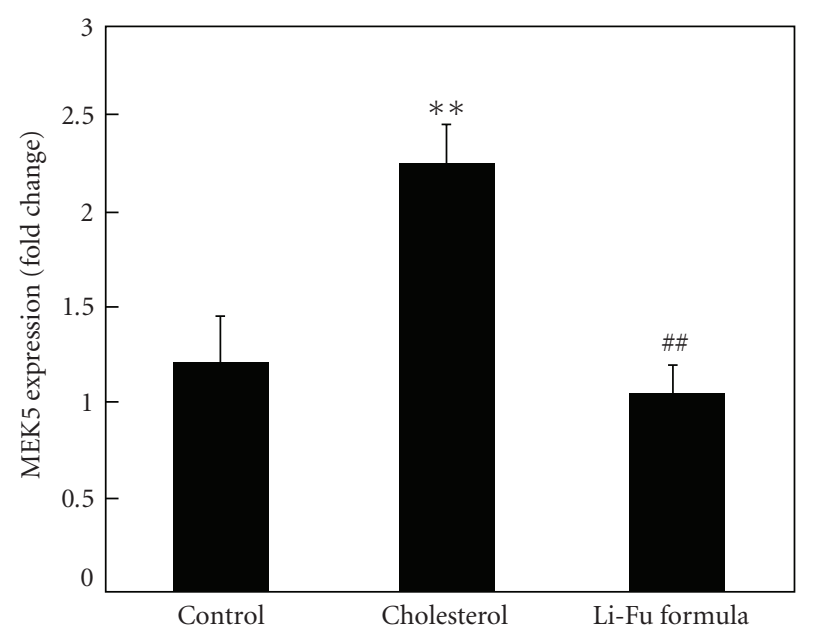

(d)

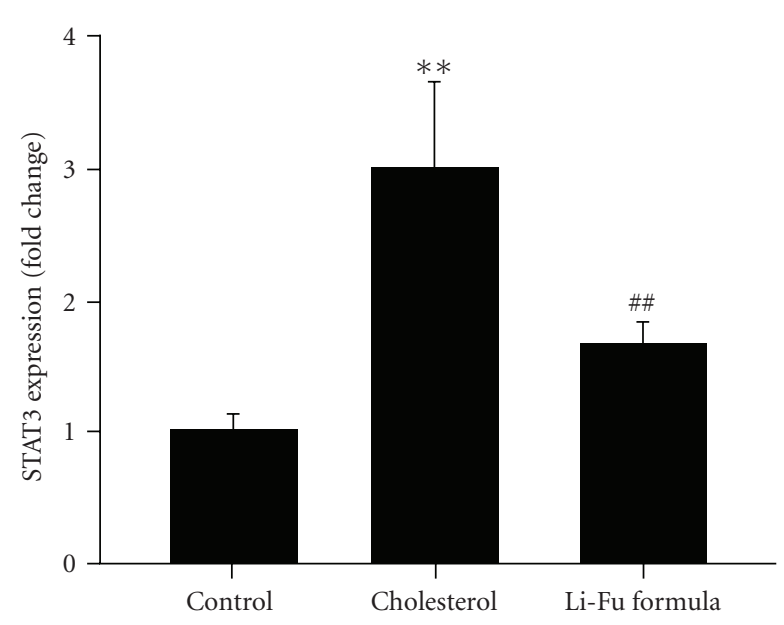

(c)

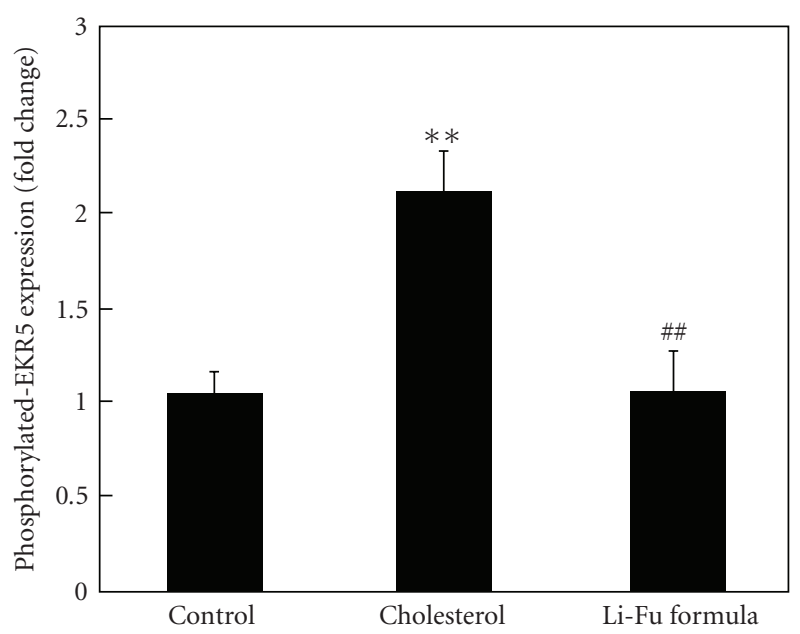

(e)

FIGURE 3: (a) The representative protein products of IL-6, STAT3, MEK5 and p-ERK5 extracted from the left ventricles of excised hearts in hamsters of control, cholesterol and Li-Fu formula groups were measured by western blotting analysis. ((b)-(e)) Bars represent the relative protein quantification of IL-6, STAT3, MEK5 and p-ERK5 on the basis of $\alpha$-tubulin. All bars indicate mean values \pm SD $(n=6$ in each group). ${ }^{* *} P<.01$, significant differences between control and cholesterol groups. ${ }^{\# \#} P<.01$, significant differences between cholesterol and Li-Fu formula groups.

In contrast, the irregular myocardial architecture and the increased interstitial space were observed in cholesterol group that showed structural disorganization and cardiomyocyte disarray. However, these abnormal architectures were significantly decreased in Li-Fu formula group compared with cholesterol group (Figure 1(b)). Moreover, the protein levels of both ANP and BNP were significantly increased in hearts of cholesterol group compared to control group. 


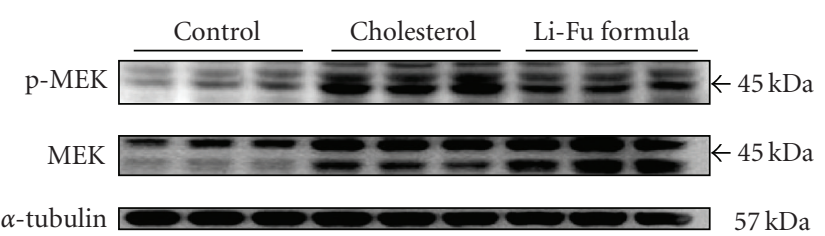

(a)

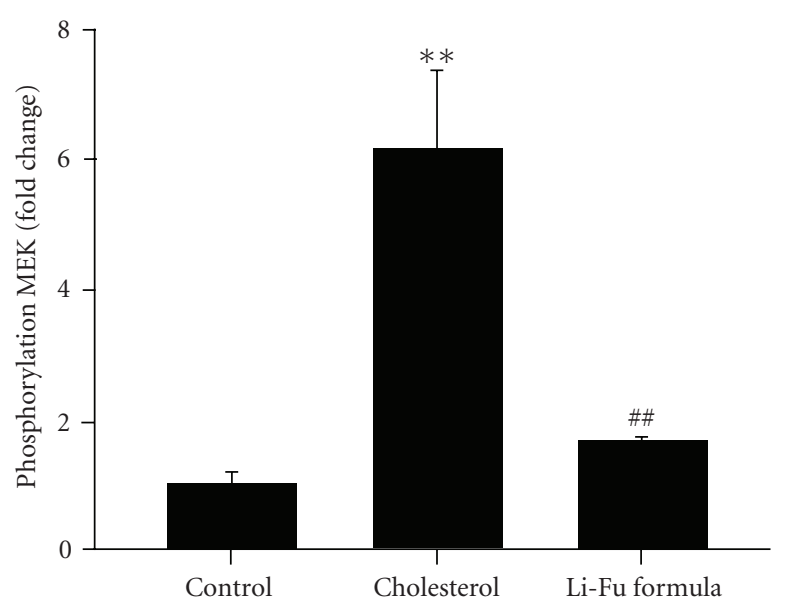

(b)

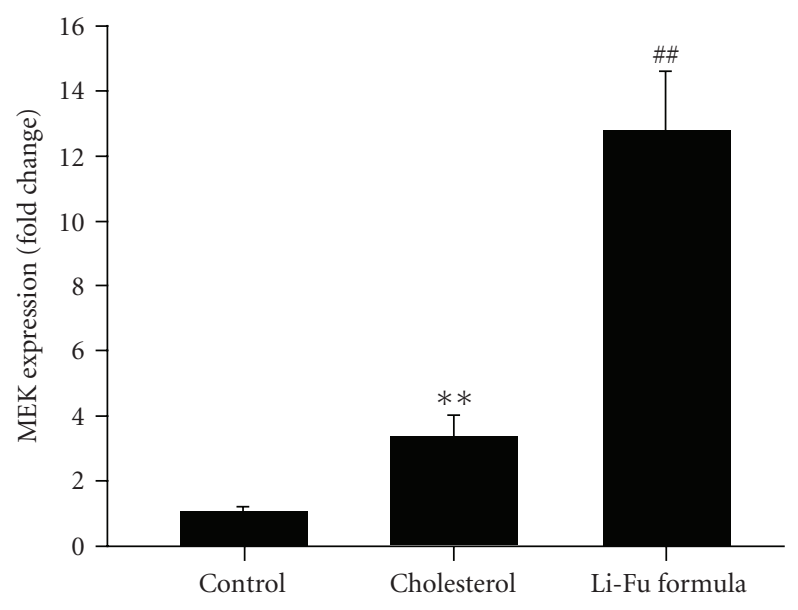

(c)

FIGURE 4: (a) The representative protein products of p-MEK and MEK extracted from the left ventricles of excised hearts in hamsters of control, cholesterol and $\mathrm{Li}-\mathrm{Fu}$ formula groups were measured by western blotting analysis. ((b), (c)) Bars represent the relative protein quantification of $\mathrm{p}$-MEK and MEK on the basis of $\alpha$ tubulin. All bars indicate mean values $\pm \mathrm{SD}$ ( $n=6$ in each group). ${ }^{* *} P<.01$, significant differences between control and cholesterol groups. ${ }^{\#} P<.01$, significant differences between cholesterol and Li-Fu formula groups.

In contrast, significantly reduced ANP and BNP protein expressions were detected in hearts of hamsters from Li-Fu formula group (Figure 2).

3.3. Effect of Li-Fu Formula on Cardiac HypertrophyAssociated Signaling Pathways. In order to identify the hyper-

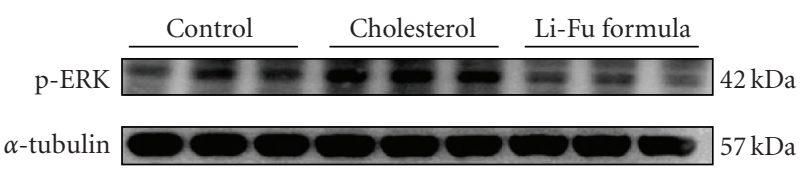

(a)

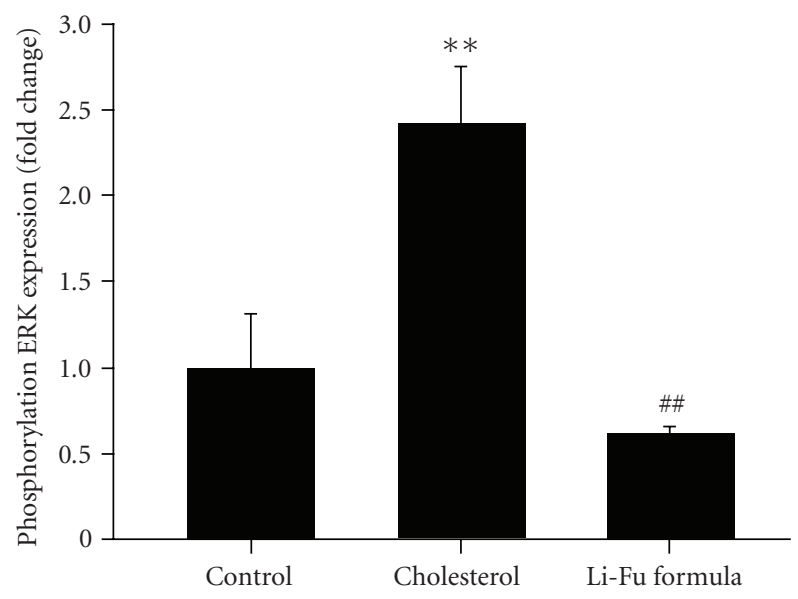

(b)

FIGURE 5: (a) The representative protein product of $p$-EKR extracted from the left ventricles of excised hearts in hamsters of control, cholesterol and Li-Fu formula groups were measured by western blotting analysis. (b) Bars represent the relative protein quantification of $\mathrm{p}$-ERK on the basis of $\alpha$-tubulin. All bars indicate mean values $\pm \mathrm{SD}$ ( $n=6$ in each group). ${ }^{* *} P<.01$, significant differences between control and cholesterol groups. ${ }^{\#} P<.01$, significant differences between cholesterol and $\mathrm{Li}-\mathrm{Fu}$ formula groups.

trophic factor IL-6, signal transducer and activator of transcription STAT-3 and mitogen-activated protein kinase/ERK (MEK) signaling pathways that were associated with the cardiac hypertrophy induced by hypercholesterol diet, the protein products of IL-6, STAT3, MEK5 and p-ERK5 were measured by western blotting. In hearts from the cholesterol group, the protein products of IL-6, STAT3, MEK5 and pERK5 were significantly increased compared to the hearts of control group (Figure 3). However, significantly decreased IL-6, STAT3, MEK5 and p-ERK5 protein expression was observed in hearts of $\mathrm{Li}-\mathrm{Fu}$ formula group (Figure 3 ). We further detected the protein levels of MEK and p-MEK. As shown in Figure 4, significantly increased MEK and p-MEK protein levels were detected in hearts of cholesterol group compared to control group (Figure 4). Notably, significantly decreased $\mathrm{p}$-MEK was observed in hearts of $\mathrm{Li}-\mathrm{Fu}$ formula group compared to cholesterol group (Figure 4). Additionally, significantly increased $\mathrm{p}$-ERK protein was detected in hearts of cholesterol group compared to control group. In contrast, significantly reduced $\mathrm{p}$-ERK protein was observed in hearts of hamsters from Li-Fu formula group compared to those from cholesterol group (Figure 5). However, no significant variations in p-P38 and p-JNK protein levels were detected among all experimental groups (data not shown). 


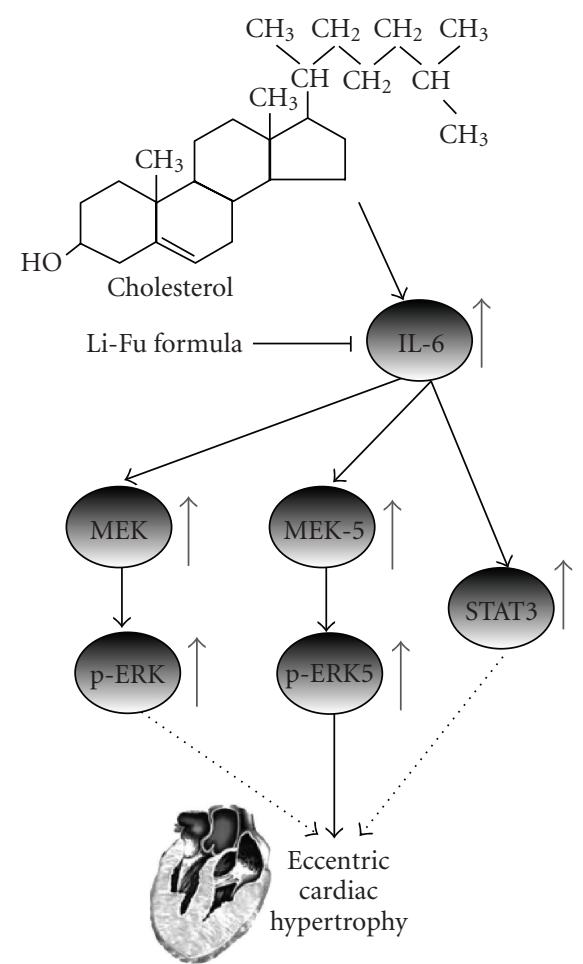

FIgURe 6: Our proposed hypothesis that cardiac IL-6, MEK-5ERK-5 and STAT3 hypertrophic pathways and MEK1/2-ERK1/2 non-cardiacmyocyte proliferative pathway are more activated in hyper cholesterol-fed hamster hearts. The eccentric hypertrophyrelated pathway, IL-6-related MEK5-ERK5 pathways and MEK1/2ERK1/2 non-cardiacmyocyte proliferative pathway may play a part of role for developing eccentric cardiac hypertrophy and pathological changes in hyper cholesterol-fed hamster hearts. Dash lines represent possible theoretical pathways but are still unconfirmed. Up arrows and down arrows on the right side represent increases and decreases, respectively.

\section{Discussion}

Hypercholesterolemia diets have been recognized as the major cause of cardiac hypertrophy and are associated with various heart diseases $[4,5,7,32]$. Our experimental results indicate the significant reduction of the WHW/BW and $\mathrm{LVW} / \mathrm{BW}$ ratios in hamsters from $\mathrm{Li}-\mathrm{Fu}$ formula group compared with those from cholesterol group. Moreover, the hypertrophic marker protein such as ANP, BNP, eccentric hypertrophic-related factors such as IL-6, STAT3, MEK5, pERK5, p-MEK and p-ERK were significantly increased in cholesterol group, whereas significant reduction of all these proteins were observed in $\mathrm{Li}$-Fu formula group.

The interleukin (IL)-6 is known as a pleiotypic factor that has been associated with various cardiac diseases $[14,36,37]$. Elevated IL- 6 mRNA is observed in patients of cardiac hypertrophy with hypertrophic cardiomyopathy [37]. As shown in Figure 6, STAT3 and MAPK (ERK)s pathway were induced by IL- 6 receptor signaling systems and contributed to the cardiac hypertrophy $[1,9]$. Accordingly, our experimental results revealed significant elevation of IL-6 expression in the excised ventricle of hamsters from
Cholesterol group as well as those hypertrophic related signaling molecules including STAT3, MEK5, p-ERK5. Notably, the significant reduction of these hypertrophic factors and signaling molecules were detected in the excised ventricle of hamsters from Li-Fu formula group. To further clarify the involved signaling pathway, we further examined the MAPK pathway that consists three major cascades including the non-cardiomyocyte proliferative ERK, and the inflammatory related c-Jun N-terminal kinases (JNK), and the p38 MAPK cascades (p38) [24]. As revealed in current study, significant increase of p-ERK was observed in the excised ventricle of hamster from Cholesterol group and the p-ERK level was significantly reduced in the excised ventricle of hamster from $\mathrm{Li}-\mathrm{Fu}$ formula group. Moreover, higher increase of p-MEK, the upstream kinase activator of EKR, was also detected in hamsters from Li-Fu group compared to cholesterol group. However, no significant differences in protein levels of p-P38 and p-JNK were detected between hamsters from cholesterol and $\mathrm{Li}-\mathrm{Fu}$ formula groups. Although the precise mechanisms of $\mathrm{Li}-\mathrm{Fu}$ formula on reducing IL-6 expression merit further investigations, these findings indeed demonstrated that $\mathrm{Li}^{-}$ $\mathrm{Fu}$ formula has the effect against cardiac hypertrophy by attenuating non-cardiomyocyte proliferation related p-ERK cascade but not P38 or JNK cascade.

Because of the moderated side effects than Western drugs, more than half of the population in the world relies on traditional medicine for therapeutic needs. Indeed, herbal remedies and alternative medicines are used throughout the world and in the past herbs often represented the original sources of most drugs [38-40]. The Li-Fu formula was first created by Dr Li-Fu Chen, China Medical University, Taichung, Taiwan, and composed of various dietary supplements and oriental herbs, including celery, black fungus, mushroom, Saliva miltior rhiza, Crataegi cuneata and Astragali radix that were routinely used as traditional medicine in oriental worlds. For instance, a major ingredient of $\mathrm{Li}-\mathrm{Fu}$ formula, Salvia miltiorrhiza, is known as "Danshen" and mainly composed of sodium tanshinone IIA sulfonate (STS), a derivative of tanshinone IIA that is also known to protect cardiovascular ischemia-reperfusion and oxidant injuries $[15,27,28,30,32-34,39,40]$. To elucidate the effect and possible mechanism of $\mathrm{Li}$-Fu formula on hypercholesterolemia-induced cardiac hypertrophy, we performed the histopathological analysis and western blotting to measure the myocardial architecture, and expression of different cardiac hypertrophy-associated molecules in the excised left ventricle from hamsters. Notably, markedly reduced ratios of WHW/BW and LVW/BW were observed in hamsters from $\mathrm{Li}-\mathrm{Fu}$ formula group compared with those from cholesterol group. These findings did suggest the protective effects of Li-Fu formula on cardiac hypertrophy.

In the world, more than half of the population relies on traditional medicine for therapeutic needs either by stewing or solution extracting [39-41]. Although the precise mechanism of most herbal medicine or dietary supplement has not been fully understood, the experience of the traditional use over the years cannot be neglected. Altogether, our experimental results revealed that $\mathrm{Li}-\mathrm{Fu}$ formula, the traditional oriental herbs and diet supplements formula have 
significant protective effects against cardiac hypertrophy. Besides the attenuated expression of ANP and BNP, the effect against cardiac hypertrophy of $\mathrm{Li}-\mathrm{Fu}$ formula is probably via the reduction of eccentric hypertrophy related IL-6 receptor pathway and non-cardiomyocyte proliferation involved ERK signaling cascade but not JNK and P38 cascades.

\section{Funding}

Grant CMU96-100 from the China Medical University, Taichung, Taiwan.

\section{Acknowledgments}

C.-Y. Huang, T.-C. Hsu, and B.-S. Tzang contribute equally to this work.

\section{References}

[1] K. J. Harjai, "Potential new cardiovascular risk factors: left ventricular hypertrophy, homocysteine, lipoprotein(a), triglycerides, oxidative stress, and fibrinogen," Annals of Internal Medicine, vol. 131, no. 5, pp. 376-386, 1999.

[2] G. W. Dorn II and H. S. Hahn, "Genetic factors in cardiac hypertrophy," Annals of the New York Academy of Sciences, vol. 1015, pp. 225-237, 2004.

[3] T. Wakatsuki, J. Schlessinger, and E. L. Elson, "The biochemical response of the heart to hypertension and exercise," Trends in Biochemical Sciences, vol. 29, no. 11, pp. 609-617, 2004.

[4] N. S. Dhalla, A. Ziegelhoffer, P. K. Singal, V. Panagia, and K. S. Dhillon, "Subcellular changes during cardiac hypertrophy and heart failure due to bacterial endocarditis," Basic Research in Cardiology, vol. 75, pp. 81-91, 1980.

[5] A. M. Gotto Jr., "Heart disease in the assessment and treatment of hypercholesterolemia: coronary artery disease and other atherosclerotic disease, family history, and left ventricular hypertrophy," American Journal of Medicine, vol. 96, pp. 9S18S, 1994.

[6] Q. M. Chen, V. C. Tu, S. Purdon, J. Wood, and T. Dilley, "Molecular mechanisms of cardiac hypertrophy induced by toxicants," Cardiovascular Toxicology, vol. 1, no. 4, pp. 267283, 2001.

[7] S. Zhang, M. H. Picard, E. Vasile et al., "Diet-induced occlusive coronary atherosclerosis, myocardial infarction, cardiac dysfunction, and premature death in scavenger receptor class B type I-deficient, hypomorphic apolipoprotein ER61 mice," Circulation, vol. 111, no. 25, pp. 3457-3464, 2005.

[8] H. Itoh and K. Nakao, "Natriuretic peptide system," Nippon Rinsho, vol. 55, pp. 1923-1936, 1997.

[9] S. J. Cameron, S. Itoh, C. P. Baines et al., "Activation of big MAP kinase 1 (BMK1/ERK5) inhibits cardiac injury after myocardial ischemia and reperfusion," FEBS Letters, vol. 566, pp. 255-260, 2004.

[10] A. Luchner, T. L. Stevens, D. D. Borgeson et al., "Differential atrial and ventricular expression of myocardial BNP during evolution of heart failure," American Journal of Physiology, vol. 274, no. 5, pp. H1684-H1689, 1998.

[11] H. Kawakami, H. Okayama, M. Hamada, and K. Hiwada, "Alteration of atrial natriuretic peptide and brain natriuretic peptide gene expression associated with progression and regression of cardiac hypertrophy in renovascular hypertensive rats," Clinical Science, vol. 90, pp. 197-204, 1996.
[12] Y. Saito, K. Nakao, H. Arai et al., "Augmented expression of atrial natriuretic polypeptide gene in ventricle of human failing heart," Journal of Clinical Investigation, vol. 83, no. 1, pp. 298-305, 1989.

[13] B. Swynghedauw, "Molecular mechanisms of myocardial remodeling," Physiological Reviews, vol. 79, no. 1, pp. 215-262, 1999.

[14] T. Kanda and T. Takahashi, "Interleukin-6 and cardiovascular diseases," Japanese Heart Journal, vol. 45, no. 2, pp. 183-193, 2004.

[15] K. Takahashi, X. Ouyang, K. Komatsu et al., "Sodium tanshinone IIA sulfonate derived from Danshen (Salvia miltiorrhiza) attenuates hypertrophy induced by angiotensin II in cultured neonatal rat cardiac cells," Biochemical Pharmacology, vol. 64, pp. 745-749, 2002.

[16] T. Hirano, "Interleukin 6 and its receptor: ten years later," International Reviews of Immunology, vol. 16, no. 3-4, pp. 249284, 1998.

[17] H. Kodama, K. Fukuda, J. Pan et al., "Leukemia inhibitory factor, a potent cardiac hypertrophic cytokine, activates the JAK/STAT pathway in rat cardiomyocytes," Circulation Research, vol. 81, no. 5, pp. 656-663, 1997.

[18] H. Hirota, K. Yoshida, T. Kishimoto, and T. Taga, "Continuous activation of gp130, a signal-transducing receptor component for interleukin 6-related cytokines, causes myocardial hypertrophy in mice," Proceedings of the National Academy of Sciences of the United States of America, vol. 92, no. 11, pp. 4862-4866, 1995.

[19] A. Ogata, N. Nishimoto, and K. Yoshizaki, "Advances in interleukin-6 therapy," Rinsho Byori, vol. 47, no. 4, pp. 321326, 1999.

[20] K. Kunisada, E. Tone, Y. Fujio, H. Matsui, K. YamauchiTakihara, and T. Kishimoto, "Activation of gp130 transduces hypertrophic signals via STAT3 in cardiac myocytes," Circulation, vol. 98, no. 4, pp. 346-352, 1998.

[21] D. C. H. Ng, N. W. Court, C. G. Dos Remedios, and M. A. Bogoyevitch, "Activation of signal transducer and activator of transcription (STAT) pathways in failing human hearts," Cardiovascular Research, vol. 57, no. 2, pp. 333-346, 2003.

[22] R. L. Nicol, N. Frey, G. Pearson, M. Cobb, J. Richardson, and E. N. Olson, "Activated MEK5 induces serial assembly of sarcomeres and eccentric cardiac hypertrophy," $E M B O$ Journal, vol. 20, no. 11, pp. 2757-2767, 2001.

[23] H. Takano, Y. Zou, H. Akazawa et al., "Inhibitory molecules in signal transduction pathways of cardiac hypertrophy," Hypertension Research, vol. 25, no. 4, pp. 491-498, 2002.

[24] C. Ruwhof and A. Van Der Laarse, "Mechanical stress-induced cardiac hypertrophy: Mechanisms and signal transduction pathways," Cardiovascular Research, vol. 47, no. 1, pp. 23-37, 2000.

[25] T. L. Wadsworth, T. L. McDonald, and D. R. Koop, "Effects of Ginkgo biloba extract (EGb 761) and quercetin on lipopolysaccharide-induced signaling pathways involved in the release of tumor necrosis factor-alpha," Biochemical Pharmacology, vol. 62, pp. 963-974, 2001.

[26] Y. K. Yim, H. Lee, K. E. Hong et al., "Anti-inflammatory and immune-regulatory effects of subcutaneous Perillae fructus extract injections on OVA-induced asthma in mice," EvidenceBased Complementary and Alternative Medicine, vol. 7, pp. 7986,2010

[27] T. W. Wu, L. H. Zeng, K. P. Fung et al., "Effect of sodium tanshinone IIA sulfonate in the rabbit myocardium and on human cardiomyocytes and vascular endothelial cells," Biochemical Pharmacology, vol. 46, pp. 2327-2332, 1993. 
[28] L. Yang, X. Zou, Q. Liang et al., "Sodium tanshinone IIA sulfonate depresses angiotensin II-induced cardiomyocyte hypertrophy through MEK/ERK pathway," Experimental and Molecular Medicine, vol. 39, pp. 65-73, 2007.

[29] L. W. Qi, Q. T. Yu, P. Li et al., "Quality evaluation of Radix Astragali through a simultaneous determination of six major active isoflavonoids and four main saponins by highperformance liquid chromatography coupled with diode array and evaporative light scattering detectors," The Journal of Chromatography A, vol. 1134, pp. 162-169, 2006.

[30] Y. H. Shon, J. H. Kim, and K. S. Nam, "Effect of Astragali radix extract on lipopolysaccharide-induced inflammation in human amnion," Biological \& Pharmaceutical Bulletin, vol. 25, no. 1, pp. 77-80, 2002.

[31] I. M. Liu, T. F. Tzeng, and S. S. Liou, "A Chinese herbal decoction, Dang Gui Bu Xue Tang, prepared from Radix Astragali and Radix Angelicae sinensis, Ameliorates insulin resistance induced by a high-fructose diet in rats," EvidenceBased Complementary and Alternative Medicine, 2009.

[32] X.-J. Chen, Z.-P. Bian, S. Lu et al., "Cardiac protective effect of Astragalus on viral myocarditis mice: comparison with Perindopril," American Journal of Chinese Medicine, vol. 34, no. 3, pp. 493-502, 2006.

[33] K. Takahashi, X. Ouyang, K. Komatsu et al., "Sodium tanshinone IIA sulfonate derived from Danshen (Salvia miltiorrhiza) attenuates hypertrophy induced by angiotensin II in cultured neonatal rat cardiac cells," Biochemical Pharmacology, vol. 64, pp. 745-749, 2002.

[34] J. D. Adams Jr., M. Wall, and C. Garcia, "Salvia columbariae contains tanshinones," Evidence-Based Complementary and Alternative Medicine, vol. 2, no. 1, pp. 107-110, 2005.

[35] W.-W. Kuo, T.-C. Hsu, M.-H. Chain et al., "Attenuated cardiac mitochondrial-dependent apoptotic effects by Li-Fu formula in hamsters fed with a hypercholesterol diet," Evidence-Based Complementary and Alternative Medicine, 2009.

[36] N. P. Visavadiya and A. V. R. L. Narasimhacharya, "Asparagus root regulates cholesterol metabolism and improves antioxidant status in hypercholesteremic rats," Evidence-Based Complementary and Alternative Medicine, vol. 6, no. 2, pp. 219-226, 2009.

[37] A. Vojdani, J. Lambert, and G. Kellermann, "The role of Th17 in neuroimmune disorders: a target for CAM therapy. Part III," Evidence-Based Complementary and Alternative Medicine, 2009.

[38] R. Patel, D.-S. Lim, D. Reddy et al., "Variants of trophic factors and expression of cardiac hypertrophy in patients with hypertrophic cardiomyopathy," Journal of Molecular and Cellular Cardiology, vol. 32, no. 12, pp. 2369-2377, 2000.

[39] C. D. Egan, "Addressing use of herbal medicine in the primary care setting," Journal of the American Academy of Nurse Practitioners, vol. 14, no. 4, pp. 166-171, 2002.

[40] E. L. Cooper, "Drug discovery, CAM and natural products," Evidence-Based Complementary and Alternative Medicine, vol. 1, pp. 215-217, 2004.

[41] E. L. Cooper, "CAM, eCAM, bioprospecting: the 21st century pyramid," Evidence-Based Complementary and Alternative Medicine, vol. 2, no. 2, pp. 125-127, 2005. 


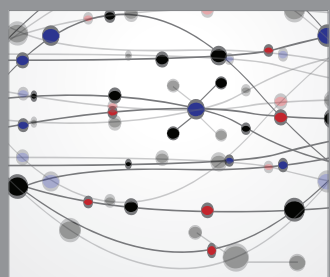

The Scientific World Journal
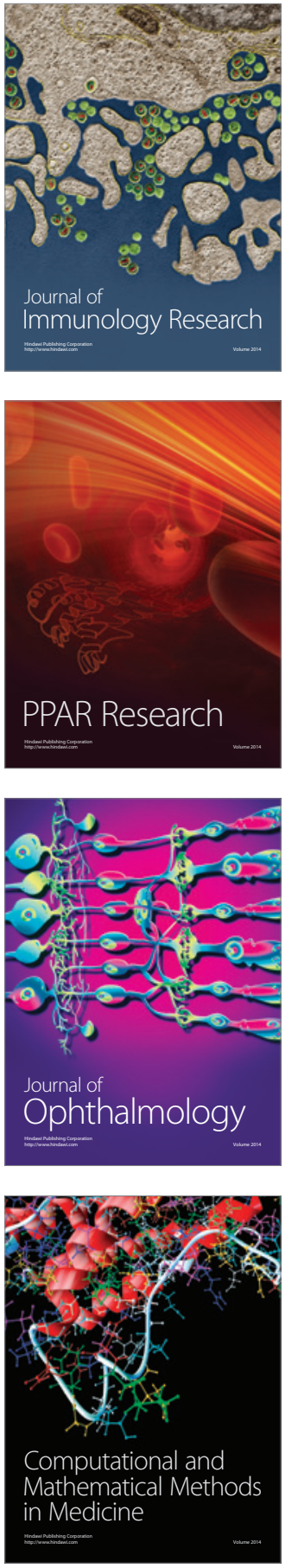

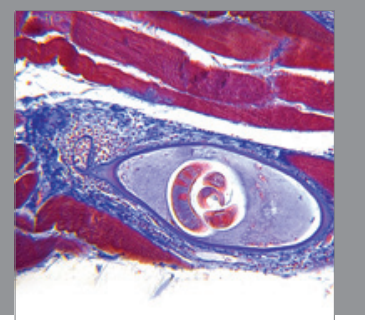

Gastroenterology

Research and Practice
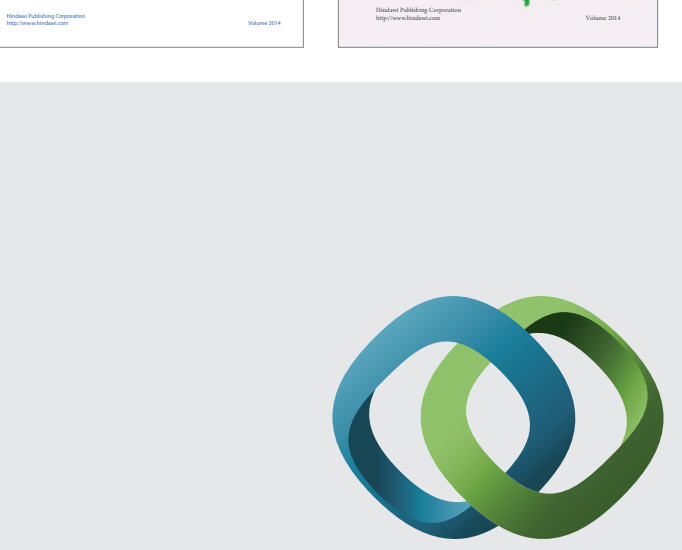

\section{Hindawi}

Submit your manuscripts at

http://www.hindawi.com
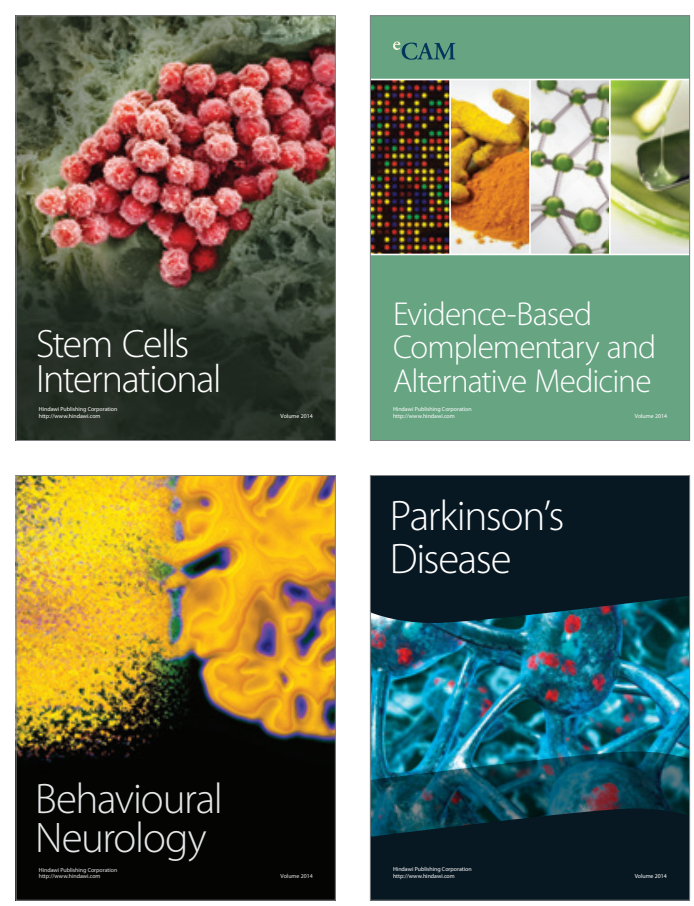

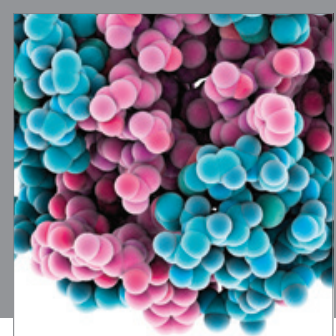

Journal of
Diabetes Research

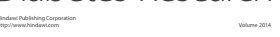

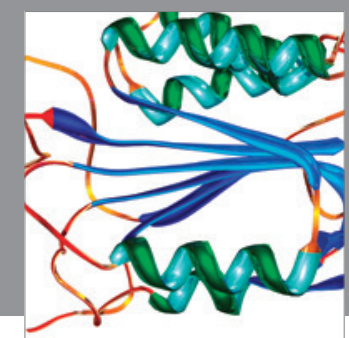

Disease Markers
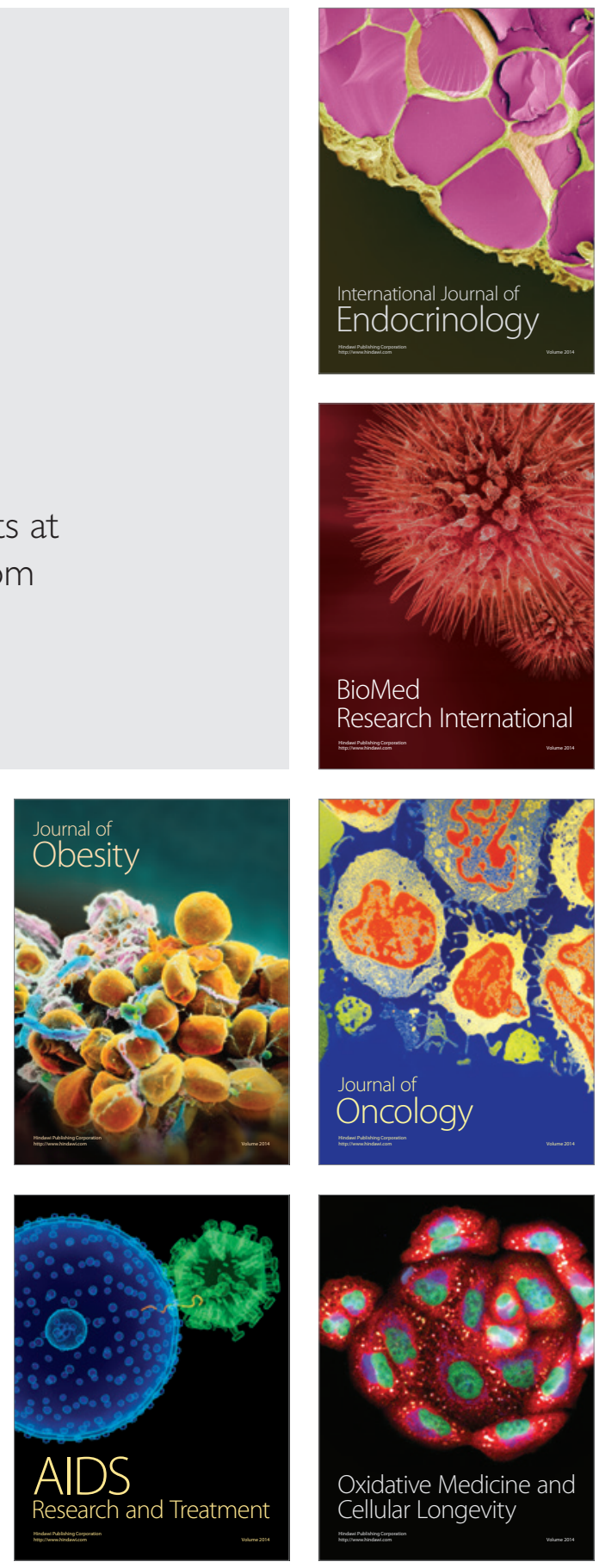